\title{
To the New Concept of Green Tribology
}

\author{
Vladimir A. Levchenko, Ilia A. Buyanovskii², Vladimir N. Matveenko ${ }^{1}$ \\ ${ }^{1}$ Lomonosov Moscow State University, Leninskii Gory, Moscow, Russia \\ ${ }^{2}$ Institute of Engineering Science, RAS, M. Kharitonievsky, Moscow, Russia \\ Email: vladlev@mail.ru
}

How to cite this paper: Levchenko, V.A., Buyanovskii, I.A. and Matveenko, V.N. (2017) To the New Concept of Green Tribology. Journal of Materials Science and Chemical Engineering, 5, 175-187. http://dx.doi.org/10.4236/msce.2017.51021

Received: January 3, 2017

Accepted: January 22, 2017

Published: January 25, 2017

\begin{abstract}
The aim of the present work is to confirm the fundamental possibility of creating new generation of the nanostructured composite coatings-orientants are studied under conditions of boundary lubrication in inactive oil, as green tribology aspect and to experimentally prove the efficacy of the nanostructured composite coatings-orientants for engineering applications. Tribological properties of nanostructured composite coatings-orientants are studied under conditions of boundary lubrication in inactive oil, as green tribology aspect. The friction tests were carried out by using two test configurations: "ball-ondisc" and "ring-to-ring". As lubricants some model and commercial oils were used. It is found that the friction coefficient and its temperature dependence differ significantly for nanostructured composite coatings under study. The obtained results were attributed to different orientating effect of these coatings on structural ordering in boundary layers, which structure is considered as a mesophase of liquid crystals. The findings suggest that the nanostructured composite coatings-orientants with orientating effect on boundary layers are advantageous for improving antifriction characteristics and for governing processes of boundary lubrication. The nanostructured composite coatingsorientants can improve lubricating properties of oils (with and without additives) and may be advantageous for engineering practice as they improve antifriction characteristics of rubbing pairs and allow controlling the processes of boundary lubrication. Thus, received nanostructured composite coatingsorientants can be today the best materials of green tribology in engineering.
\end{abstract}

\section{Keywords}

Green Tribology, DLC Coatings-Orientant, Nanostructural Coating, Boundary Lubricant Layers, Structural Order

\section{Introduction}

Our understanding of mechanism of lubricating action of boundary layers can 
be essentially extended on the base of multidisciplinary approach to the problem. Recently, the concept of "green tribology" [1] regarding as "the science and technology of the tribological aspects of ecological balance and of environmental and biological impacts" was introduced. The field of green tribology includes tribological technology that mimics living nature (biomimetic surfaces) and thus is expected to be environmentally friendly, the control of friction and wear that is of importance for energy conservation and conversion, environmental aspects of lubrication and surface modification techniques, and tribological aspects of green applications such as wind-power turbines or another tribological devices. This paper is the first comprehensive volume on green tribology with using coating-orientants. In the presented paper, lubricating properties of boundary layers were considered from the position of tribology, nanotechnology and some concepts of physics and chemistry of liquid crystals. According to Levchenko et al. [2] [3] [4], the lubricating ability of oils depends on their capacity to form highly oriented and structurally ordered layers. It is determined both by properties of oils and that of the contacting surfaces. Active components of a lubricating medium interact with the surface and form adsorbed boundary layers. Hardy [2] suggested the boundary phase distributed as a thin lubricating film on a solid surface may be considered as a mesophase of ELC (epitropic liquid crystal). Epitropic liquid crystals are boundary (wall adjacent) hydrocarbons liquid layers, the organization and properties of which differ from traditional nematic liquid crystals [5] by its structural S-order parameter, space limitedness and formation mechanism. ELC are under the joint action of anisotropic intermolecular interaction, the fields both of the long range surface forces from solid substrate and the short-range surface forces that are determined by the concentration and type of active centers on solid surface and surface microdefects, boundaries of crystallites, surface dislocations etc. [6]. The degree of orientation of boundary layers defines their lubricating ability [4] [6] [7] [8] [9] [10], and depends on surface morphology. Thus, for obtaining high lubricating ability it is necessary to optimize parameters responsible for a boundary layer orientation both related to contacting surfaces and lube oils.

The level of molecule orientation in boundary layers can be increased in the presence of some surface coatings-orientants, used in liquid crystal technology [9] [10]. These coatings have a strong influence on the structural ordering of a boundary layer and facilitate formation of highly oriented layers of molecules in these layers. Such coatings-orientants can create planar (parallel), homeotropic (normal) and inclined orientation [10] [11] [12] of molecules in a boundary layer in respect to friction surface. According to Levchenko et al. [7] [8] [9] [10], the coatings with homeotropically orienting structure have stronger effect on the orientation ordering in boundary layers as compared to others. Such a structure can provide an optimal lubricating ability of oils. Various homeotropic orientants (lecithin, quartz and carbon) differ in their effect on the parameter ordering of the boundary layer orientation. The value of this ordering parameter usually varies from 0.1 to 0.6 [6]. The maximum ordering is provided by mono- 
crystalline carbon [12].

The present paper studies behavior of monocrystalline carbon coatingsorientants deposited on steel surfaces in comparison with amorphous nonorienting ones. The effect of these coatings on the possibility of molecular orientation and structural order changes in boundary lubricating layers is considered.

\section{Materials and Methods}

Monocrystalline carbon coatings. The monocrystalline diamond-like coating was originally prepared by the ion-assisted deposition method [10]. The basic idea was to use ion-assisted epitaxial condensation of carbon clusters on orienting surfaces to prepare ordered layers of linear carbon, while the carbon clusters are produced by ion sputtering of graphite. The mass-spectroscopic (SIMS) studies showed that carbon flux obtained by ion sputtering of a graphite target with a low-energy ion beam $(E=5 \mathrm{keV})$ consists of linear carbon chains $C_{n}$ with $n=$ $1,2, \cdots, 8$. This follows from the specific intensity distribution of carbon clusters during ion sputtering of graphite. As established by SIMS, the concentration of linear carbon clusters $C_{n}$ in a carbon vapour varies periodically as a function of the cluster size with a period $\mathrm{n}=2$, which is in agreement with the dependence of the linear carbon chain stability on $\mathrm{n}$ as predicted theoretically [1]. During the condensation, the Ar+ ion beam irradiating the growing carbon film has energy of $150 \mathrm{eV}$, which corresponds to the maximum cross-section for neutralization of Ar+ ions on the carbon chain-like clusters. This situation is favorable for low temperature deposition of a single-crystal film in the temperature range $50^{\circ} \mathrm{C}$ $180^{\circ} \mathrm{C}$ that prevents the formation of inter-chain cross-links.

The following parameters are normally used to prepare oriented linear-chain carbon [12] a deposition rate of $1 \mathrm{~nm} / \mathrm{min}$; an ion current density of the beam irradiating the growing film of $1 \mathrm{~mA} / \mathrm{cm}^{2}$; and a residual gas pressure of $\mathrm{P}=10^{-7}$ Torr. High-purity graphite (99.99\%) is used as a target. The thickness of films was of $3 \mu \mathrm{k}$.

The atomic structure of prepared films was determined by TEM. Representative electron diffraction patterns of oriented carbon films are shown in Figure 1.

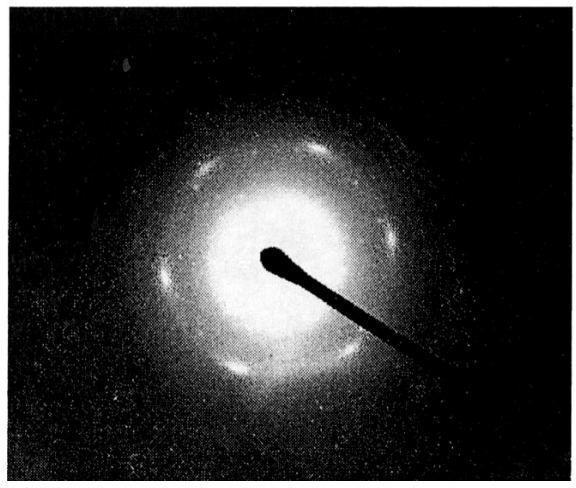

Figure 1. Electron diffraction pattern of a monocrystalline carbon coating. 
This diffraction pattern consists of six bright reflections located on the same ring. The given electron pattern proves monocrystalline structure of the film [13] [14].

The absence of higher-order reflections in the diffraction pattern is accounted for by a layered structure of the film and by small random displacements of the layers constituting the film. It should be noted that similar diffraction patterns are characteristic for multilayered films prepared by the LB (Longmuir-Blodgett) method, in which the chain axes are oriented normally to the surface of the film. This normal orientation of carbon chains can also be proven by tilting a diagnostic electron beam relative to the direction perpendicular to the film surface. In this case only two reflections can be observed in the diffraction pattern located parallel to the tilting axis. Such a pattern corresponds to the diffraction by parallel chains. The films have been distinguished due to their specific atomic and electronic structure that was studied by various spectroscopic methods.

Carbon coatings studied in the present paper were produced in the form of a monocrystalline film with two-dimensionally ordered homeotropic (perpendicular to surface) structure. In these films, there are the short, straight linear carbon chains forming monolayers. The distance between carbon chains can be calculated from diffraction pattern (Figure 1) as $\mathrm{a}=\mathrm{d}_{100} \times 2 / \sqrt{3}=4.93 \mathrm{~A}$, which well corresponds to Van der Walls diameter of carbon atoms. The chains are organized into densely packed hexagonal structures with the distance between chains of $4.8-5.03 \mathrm{~A}$. The long axis of the linear carbon chains is oriented perpendicular to the plane of the film. The carbon chains are the main structural element of the carbon film under study.

According to Auger spectroscopy data the carbon content in the films is about 99\% (remain is oxygen and nitrogen). The density of electronic states in the valence band corresponds to the calculated spectrum of linear carbon chains. The DOVS (density of valence state) was calculated from the experimental Auger spectrum of carbon films by self-deconvolution of the carbon KVV line (Figure 2).

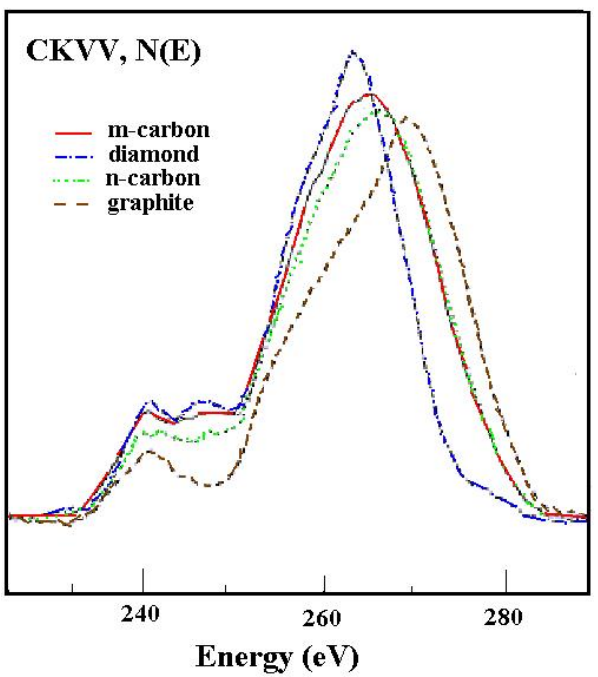

Figure 2. Auger spectra carbon films. 
The electronic terms of linear carbon chains $C_{n}$ calculated by the linear-combination-of-atomic-orbital method for $2,3, \cdots, 8$ show that when the number of carbon atoms in the chain is greater than 8 , the width of a and $n$ subbands does not change. The edges of $\mathrm{n}$ and a bands become 10, 16.5 and 18, $26 \mathrm{eV}$, respectively.

The maxima of the experimental DOVS curves at $10 \mathrm{eV}$ and $16 \mathrm{eV}$ correspond to the top and the bottom of the T-subband, and the maxima at $19 \mathrm{eV}$ and $26 \mathrm{eV}$ correspond to the top and the bottom of a-subband. Thus, Auger spectroscopy confirms the linear cumulene structure of carbon monocrystalline films.

The structure of the films was also investigated by Raman spectroscopy with argon laser excitation $(\lambda=632.8 \mathrm{~nm})$. The Raman spectrums of linear-chain carbon films with different thickness are shown in Figure 3. As one can see, when thickness of coating is $100 \mathrm{~nm}$ there is only one wide band with a maximum between 1900 and $2500 \mathrm{~cm}^{-1}$. This is typical for the vibration frequencies carbon skeleton of the chains $\mathrm{sp}^{1}$.

Using harmonic approximation this frequency can be calculated as

$$
\omega_{0}=\sqrt{\frac{2 k}{m}}
$$

where, $k$ is elastic constant $\mathrm{sp}^{1}$-bond, $\mathrm{m}$-carbon atom mass. At coating thickness $200 \mathrm{~nm}$ the second band with the maximum $1523 \mathrm{~cm}^{-1}$ appears. It can be related to regular zigzags in carbon chains. At coating thickness $300 \mathrm{~nm}$ and more Raman spectrum does not change. There is some displacement of carbon chains in the long valence bonds direction (chain length direction) when the transition from one monolayer to another occurs. The angle between these bonds is about $180^{\circ}$, as it may be seen from Figure 4 . The dangling bonds on the ends of carbon

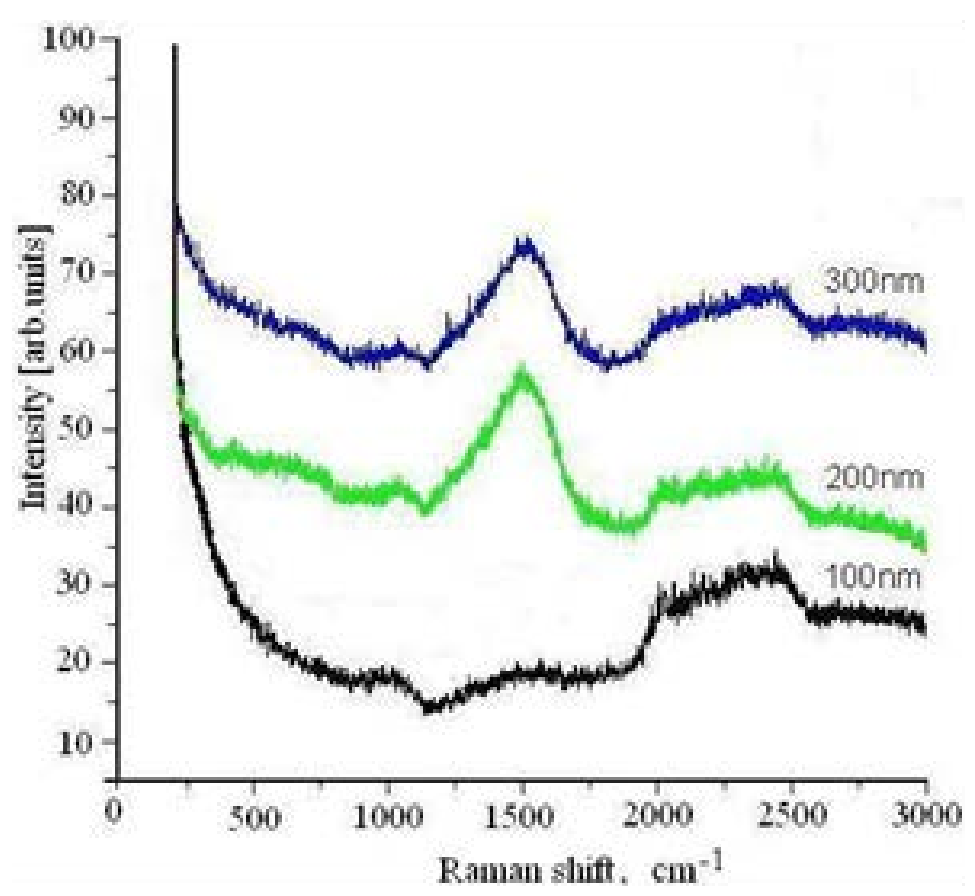

Figure 3. Raman spectrum of a linear-chain carbon coating. 


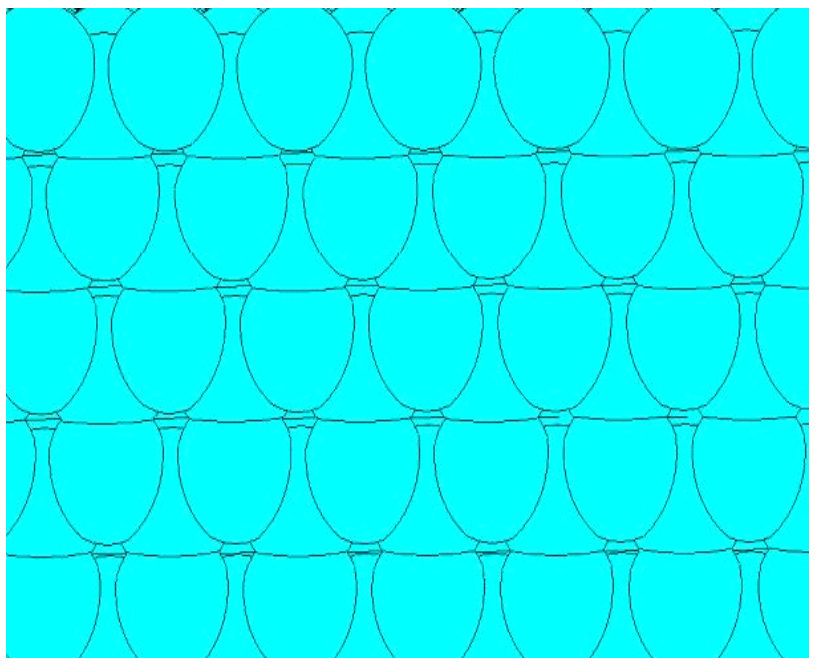

Figure 4. Atomic structure model of carbon coating.

chains may be saturated by $\mathrm{O}, \mathrm{N}, \mathrm{OH}, \mathrm{NHx}$ groups, which may interact with oil molecules by hydrogen and ionic bonds and week Van der Waals forces.

During the deposition carbon chains are oriented homeotropically (perpendicularly) to the surface of substrate and have a good adhesion to the surface. The hardness of carbon films varies from 6000 - $9800 \mathrm{HV}$. The model of the atomic crystal structure of the oriented carbon films (see Figure 4) can be presented as follows. The film is composed of parallel linear carbon chains densely packed into a hexagonal lattice. It has multilayer structure, where the layers are separated from each by regularly placed kinks in the carbon chains skeleton. The thickness of carbon films used in the present work was of $3 \mu \mathrm{m}$.

Amorphous carbon coatings. For comparison films with another type of structure, namely amorphous diamond-like ones of the same thickness $(3 \mu \mathrm{km})$ were studied, which are widely used in tribo engineering. Amorphous diamond-like films were obtained by carbon plasma impulse condensation $(\tau=400 \mu \mathrm{s}, \omega=3$ $\mathrm{Hz}, \rho=10^{13} \mathrm{~cm}^{-3}$, the level of ionization is $\left.95 \%\right)$. Due to specific distribution of potential energy the discharge of carbon ions in plasma near substrate can change in the ranges $10-80 \mathrm{eV}$ at changes in voltage $150-360 \mathrm{~V}$. Electron diffraction pattern of amorphous diamond-like coating is given in Figure 5. The thickness of amorphous films is in the ranges from $1 \mathrm{~nm}$ to $1 \mu \mathrm{m}$, the hardness up to $8000 \mathrm{HV}$ (at the atoms of carbon energy $60 \mathrm{eV}$ ). The film has also a good adhesion to substrate surface.

Lubricants. As model lubricants, the following materials were used: liquid paraffin (viscosity $v_{50}=32 \mathrm{~mm}^{2} / \mathrm{s}, v_{100}=8 \mathrm{~mm}^{2} / \mathrm{s}$, density- $880 \mathrm{~kg} / \mathrm{m}^{3}$ ) with $0.1 \%$ stearic acid or $1 \%$ oleic acids.

The additives of oleic and stearic acids were chosen as they can form a liquid crystal phase in boundary layers. These fatty acids are able also to activate the processes of orienting and structural ordering in the boundary layer of lube oil in the presence of a surface-orientant. As a base mineral oil with viscosity corresponding to SAE 30 without additives was used (viscosity $v_{50}=11.5 \mathrm{~mm}^{2} / \mathrm{s}, v_{100}=$ $10.5 \mathrm{~mm}^{2} / \mathrm{s}$, density- $900 \mathrm{~kg} / \mathrm{m}^{3}$ ). 


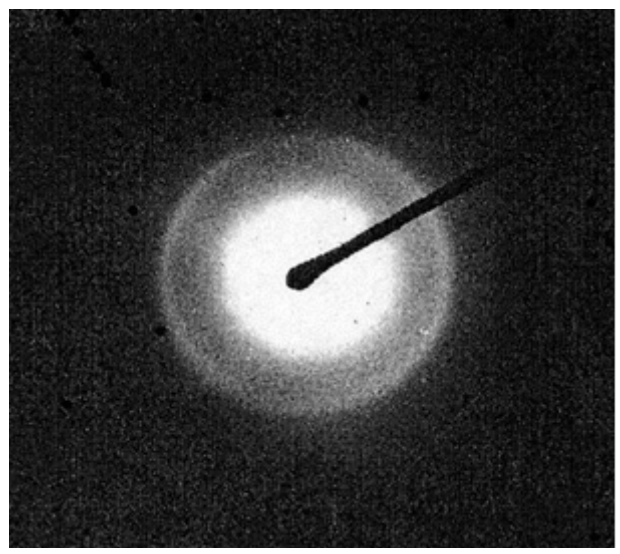

Figure 5. Electron diffraction pattern of a amorphous DLC coating.

Test Procedure. Tribological characteristics of coatings under study were evaluated by using two types of testing devices: a ball-to-disk tribometer and a tribometer where two specimens-rings were axially loaded against each other. In the first type of tests two model lubricating compositions (liquid paraffin with $1 \%$ of oleic acid and liquid paraffin with $0.1 \%$ of stearic acid) were used, whereas in the second one-a base mineral oil with viscosity corresponding to SAE 30 . The ball-to Pin-disc tribometer scheme is shown in Figure 6. The steel ball 3 with the diameter of $12.7 \mathrm{~mm}$ was sliding on the rotating steel disk (the diameter- $6 \times 10^{-2}$ $\mathrm{m}$, the thickness- $0.4 \times 10^{-2} \mathrm{~m}$ ) placed in an oil bath, the temperature of which was regulated by a special heater.

The roughness parameter $R_{a}$ of disk friction surface is $0.05-0.07 \mu \mathrm{m}$. Both the ball and the disk are made of hardened ball-bearing steel $(1.30 \%-1.65 \% \mathrm{Cr}$; 1.0\% C, Mn-0.20 - 0.40, Fe-remain). Micro hardness of balls was $920-960 \mathrm{HV}$ and that of disks-880 - $930 \mathrm{HV}$. The coatings were deposited both on the disc and ball surfaces. The specimens were tested under constant sliding speed $\mathrm{V}=$ $0.01 \mathrm{~mm} / \mathrm{s}$ and constant load $\mathrm{P}=8 \mathrm{~N}$. The oil bath temperature was increasing with the rate of $10^{\circ} \mathrm{C} / \mathrm{min}$. The tests of discs and balls with and without coatings were performed in the range of oil bath temperatures $20^{\circ} \mathrm{C}-250^{\circ} \mathrm{C}$.

The second stage of tests was performed on the friction machine UMT-1 using a friction heat stability test. The friction machines UMT-1 are shown in Figure 7.

The ring specimens made of carbon steel $(0.4 \% \mathrm{C})$ had the following sizes: outer diameter $\mathrm{D}_{1}=0.028 \mathrm{~m}$; inner diameter $\mathrm{D}_{2}=0.020 \mathrm{~m}$; height $\mathrm{h}=0.015 \mathrm{~m}$. Brinell hardness of the specimens was of $350 \mathrm{HB}$.

The specimens are axially loaded against each other. One of the specimens is rotating, whereas the other with coated face surface is restrained from motion. A thin layer of the oil SAE 30 is introduced into interfacial gap of the rubbing surfaces providing boundary lubrication. Friction torque and temperature generated due to friction are measured during tests. The temperature is measured by the $\mathrm{Cr}-\mathrm{Al}$ thermocouple inserted in the non-rotating coated specimen at the distance of $1 \times 10^{-3} \mathrm{~m}$ from the rubbing surface. The total constant load $\mathrm{P}$ is $210 \mathrm{~N}$, 


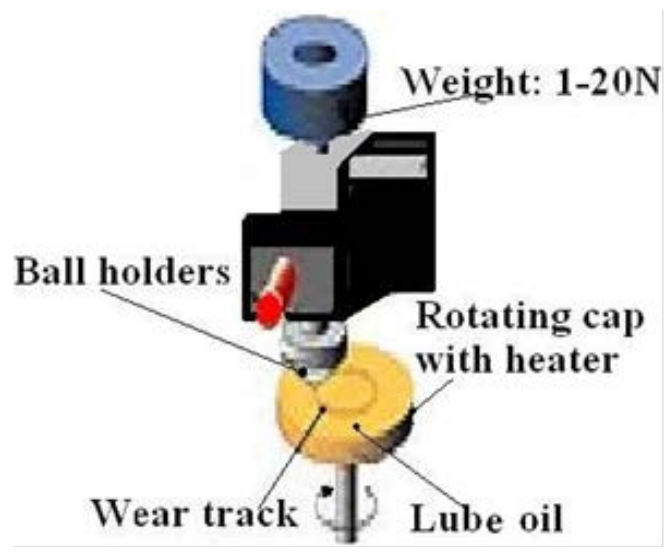

Figure 6. Pin-disc device.

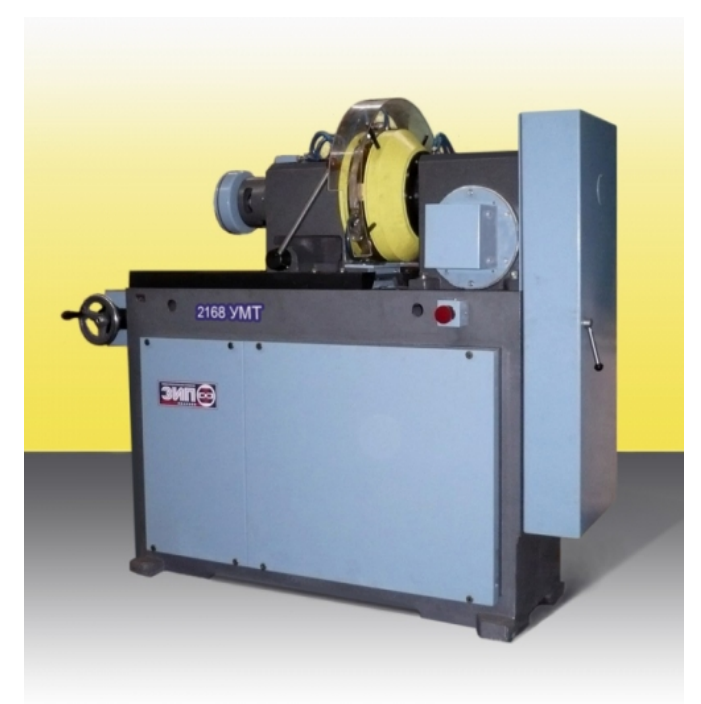

Figure 7. Friction machine UMT-1.

specific pres comma sure $\mathrm{Pa}=0.7 \mathrm{MPa}$, the speed steps are $100,200,500,700$, $1000,1500 \mathrm{rev} / \mathrm{min}(0.125-1.875 \mathrm{~m} / \mathrm{s})$. Every step of speed was lasted $30 \mathrm{~min}$ what was enough for stabilization of the contact temperatures and friction torque under constant loading conditions. Test results were the average data from three friction pairs testing.

\section{Results and Discussion}

Ball-to-disc tests. The test results of two coatings and uncoated steel discs in liquid paraffin with additives are given in Figure 8 and Figure 9. As it may be seen from the experimental data (Figure 8) the values of friction coefficient for steel without coating in liquid paraffin with stearic acid in all the range of test temperatures are higher as compared to that of specimens with carbon coatings. The minimal values of friction coefficient were obtained for the monocrystalline coating. In the range of temperature $20^{\circ} \mathrm{C}-1300^{\circ} \mathrm{C}$, its value is of 0.1 . The friction coefficient of the amorphous diamond-like coating at these temperatures is $0.16-0.14$, and that of steel is about $0.2-0.17$. There is a trend to increasing 


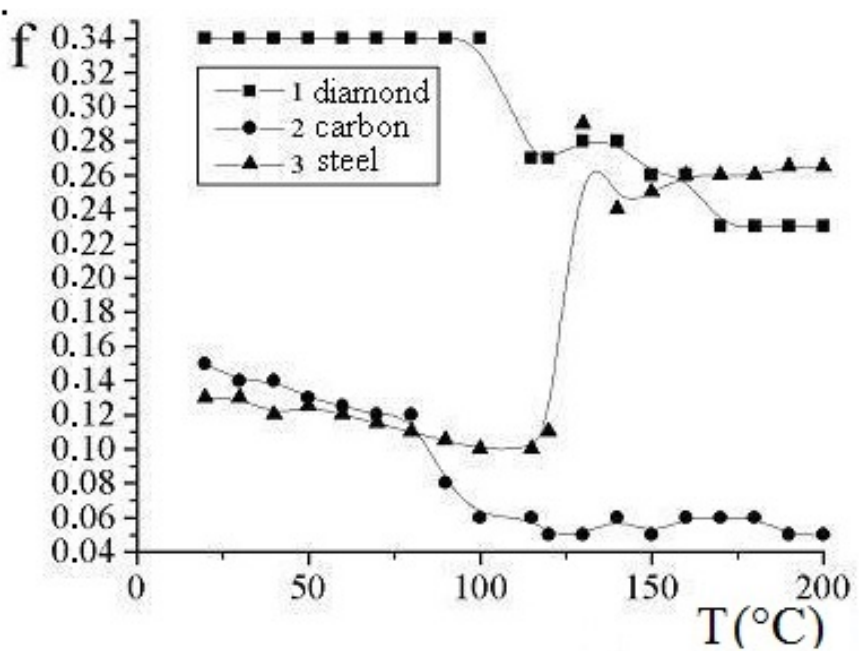

Figure 8. The friction coefficient dependence via tempera-ture in paraffin oil with $0.1 \%$ stearic acid.

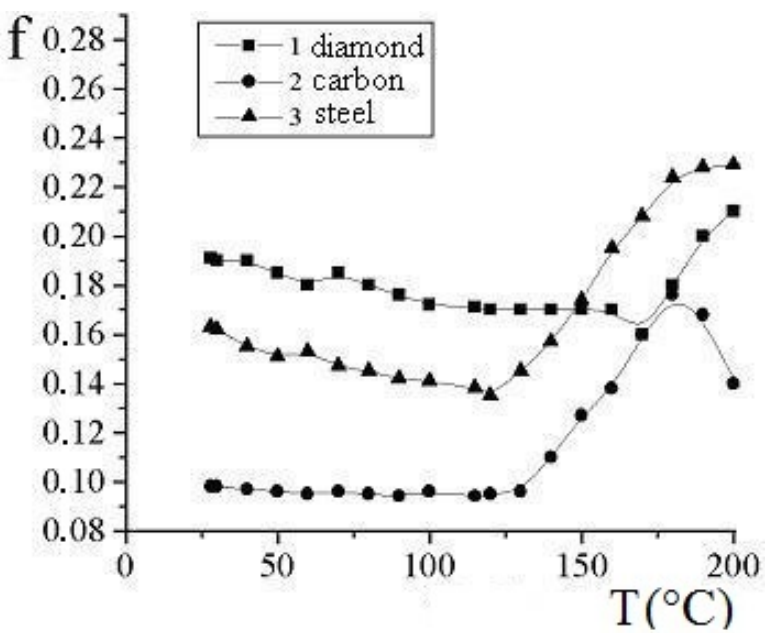

Figure 9. The friction coefficient dependence via temperature in paraffin oil with $1 \%$ oleic acid.

friction coefficient via temperature for all pairs tested in liquid paraffin with stearic acid. The sharp increase in friction coefficient occurs at so-called critical temperatures.

The value of this temperature characterizes a limit of lubricating properties of oils under boundary lubrication. The critical temperature for steel without coating is of $120^{\circ} \mathrm{C}$ (Figure 8, curve 3), whereas for steel with the monocrystalline coating this temperature is $130^{\circ} \mathrm{C}$ (Figure 8, curve 2). The amorphous diamond coating demonstrates high friction in all range of temperatures under study (Figure 8, curve 1). For uncoated surfaces and surfaces coated by monocrystalline carbon after critical temperature exceeding significant growth of friction occurs, whereas for steel with amorphous coating a trend to some decreasing friction takes place. Under tests in liquid paraffin with oleic acid, the monocrystalline carbon coating also shows the minimal friction as compared to the diamond-like amorphous coating and uncoated steel (Figure 9). The value of fric- 
tion coefficient for it is $0.12-0.04$ (Figure 9, curve 2), whereas the amorphous coating has the friction coefficient of 0.34 - 0.25 (Figure 9, curve 1) and steel$0.14-0.26$ (Figure 9, curve 3). The friction coefficient dependence via temperature in these tests differs from that what was reported above for liquid paraffin with stearic acid. We have not found here the transition to high friction with temperature increasing.

On the contrary, the tendency to friction decreasing with temperature takes place for both coatings at the temperatures exceeding $100^{\circ} \mathrm{C}$. At the same time for the uncoated steel the temperature dependence of friction coefficient is not changed. The critical temperature in this case is about $125^{\circ} \mathrm{C}$. The character of friction for uncoated steel at temperatures below critical is similar to that of the monocrystalline carbon (Figure 8, curves 2 and 3), whereas at the temperatures exceeding critical ones the steel surface behaves like to the diamond-like coating. At the temperatures above the critical one, the values of friction coefficient of steel and diamond-like coating practically coincide (Figure 8, curves 1 and 3).

Two rings tests. The second type of tests was conducted to investigate friction behavior of the carbon coatings under conditions coming nearer to real operation of tribounits. As it was mentioned above, ring specimens were made of steel $45(0.40 \% \mathrm{C})$. The coated face surface of one of the ring specimens rubbed against the uncoated steel one. As a lubricant the mineral oil corresponding to classification SAE 30 was used. Opposite to the first stage of tests the heating of specimens resulted from the heat generation due to friction. The maximum measured temperature rise during tests was up to $150^{\circ} \mathrm{C}$. After this as a rule seizure and scoring processes were intensified and the tests were interrupted.

The experimental results also show the difference in tribological behavior of carbon coatings with and without orienting properties. The reference pair "steelsteel" (Figure 10(a)) demonstrates stable friction in the range of $100-1000$
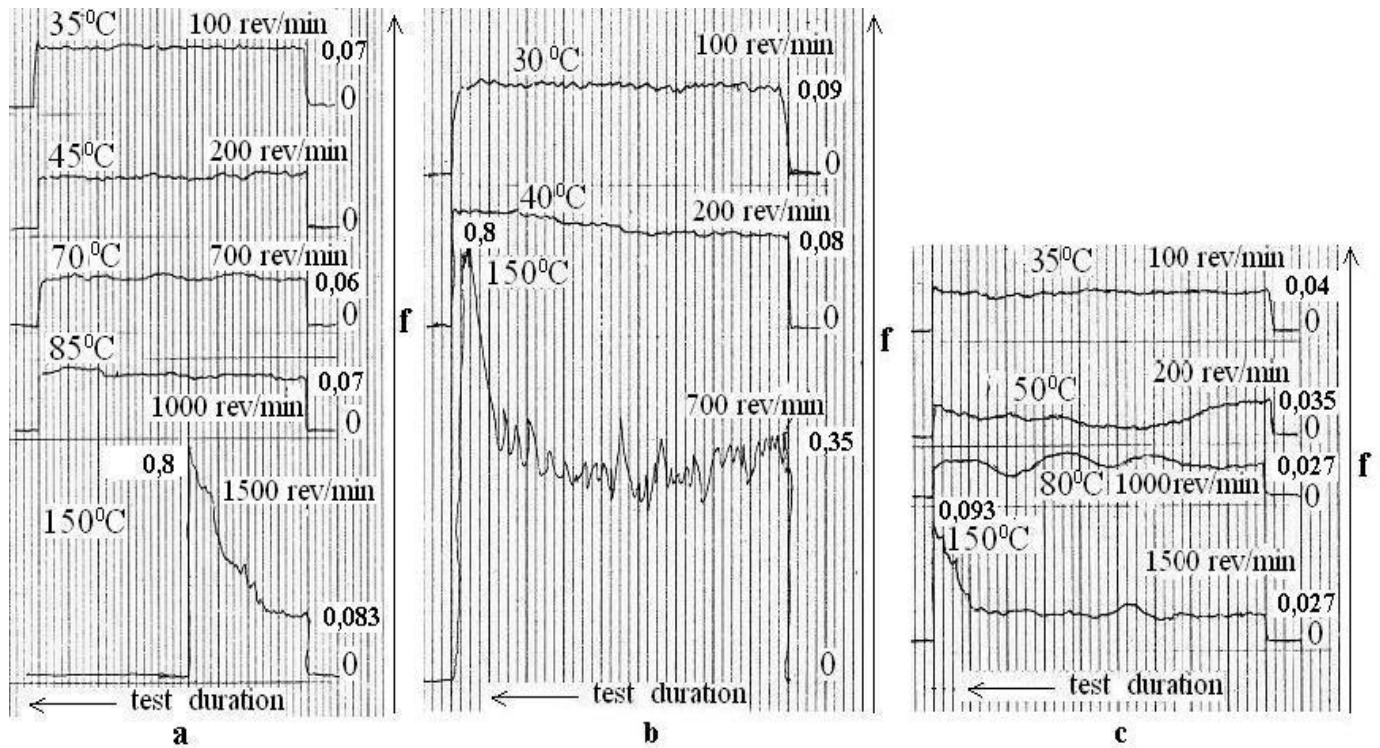

Figure 10. The friction coefficient dependence via sliding speed: (a) steel, (b) diamond-like carbon coating, (c) mono-crystalline carbon coating. 
$\mathrm{rev} / \mathrm{min}$. At $1500 \mathrm{rev} / \mathrm{min}$ (temperature is higher $125^{\circ} \mathrm{C}$ ) the scoring of uncoated specimens takes place.

The pair "uncoated steel 45-diamond-like amorphous coating" shows stable friction only at $100 \mathrm{rev} / \mathrm{min}$ and $200 \mathrm{rev} / \mathrm{min}$ (Figure 10(b)). For this pair a tendency to seizure occurs at relatively low sliding speeds. This process intensifies at $700 \mathrm{rev} / \mathrm{min}$ when temperature rises up $150^{\circ} \mathrm{C}$ and the value of friction coefficient grows up to 0.8 . The monocrystalline coating has the minimum friction coefficient $(0.016-0.093)$ in all range of sliding speeds and temperatures (Figure 10(c)).

\section{Discussion}

Basing on the conception, which considers the structure of lubricating boundary layers as mesophase of liquid crystals, the mechanism of different influence of carbon coatings on friction can be attributed to different orienting effect of these coatings on structural ordering in boundary layers. During tests in liquid paraffin both with oleic and stearic acids for the reference uncoated steel, the dependence of friction coefficient via temperature is not changed. It is possible to suggest that the lubrication mechanism of saturated and unsaturated fatty acids additives here is the same. While testing in liquid paraffin with saturated fatty acid (stearic acid) all three types of surfaces show the same friction dependence via temperature. It is possible to suggest that the formation and destruction of boundary layer in the range of temperatures up to $150^{\circ} \mathrm{C}$ occur mainly according to adsorption-desorption mechanism without breakdown of stearic acid molecules. In this case, the minimal values of friction coefficient and the best lubricating ability can be seen for the monocrystalline coating, which appears to demonstrate its orienting effect on the structural order of mesophase of epitropic liquid crystals formed by stearic acid molecules. At presented stage of investigations we consider the effect of coatings-orientants on boundary layers structuration only in the ranges of temperatures provided adsorption mechanism of boundary layers formation. If the temperatures are higher, some other mechanisms including chemisorption ones would be realized, but they need special studies and discussion. We can only mention that when unsaturated oleic acid is used as an additive to liquid paraffin friction changes via temperature for coatings under study differ essentially from the test results with stearic acid.

The additive of oleic acid seems to have an insignificant lubricating action on the diamond-like amorphous coating up to the temperatures of breakdown of binary bonds of the oleic acid molecules and their possible oxidation. Possibly it is connected with the inability of this coating to form a strongly oriented boundary layer in said lubricant in the range of temperatures up to $150^{\circ} \mathrm{C}$. The following decrease in the friction coefficient could be explained by tribochemical changes in oleic acid (for example, some monocarbon acids formation such as aselaine and pelargone ones, etc.), which may display some lubricating effect due to their ability to form a quasismectic structure i.e., epitropic liquid crystals in the boundary lubricating layer. This ELC opposite to mesophase of ELC formed 
by oleic acid probably have better ability to structural ordering. On the contrary, the monocrystalline carbon provides a significant decrease in friction in the presence of oleic acid. In our opinion, it may be explained by some changes in molecular structural order in a boundary ELC layer of the lubricant due to the orienting effect of the coating. At Figure 1 and Figure 11 the electron diffraction patterns of a monocrystalline carbon film and that with paraffin oil and oleic acid are presented. There are isolated reflections, which are typical for oriented structures (see Figure 11).

The random displacement of the oriented oil layers results in strong damping of higher order reflections in the diffraction pattern of the oriented carbon film. For the amorphous diamond-like carbon coating any preferred orientation both in the lubricating layer and surface film itself was not found (see Figure 5). The data obtained in the tests with the mineral oil also show the absence of a significant lubrication action of this oil on the amorphous diamond-like coating and as a result the tendency to seizure at low sliding speeds and temperatures. The said oil is a base one, i.e., not containing any additives. Thus we can suggest that in the case of monocrystalline coating some structural ordering of oil molecules takes place facilitating friction.

\section{Summary}

The results of experiments have shown that the values of friction coefficient and the friction dependence via temperature are determined by the type of coating and its orientation properties. The difference in friction characteristics is connected with the different degree of orientation of molecules ELC in boundary lubricating layers on rubbing surfaces. Neither steel nor amorphous diamondlike coatings are not structural orientants. On the contrary carbon monocrystalline coatings increase the degree of molecular structural ordering in the boundary lubricating layers and consequently their lubricating ability. The monocrystalline coatings-orientants can improve lubricating properties of oils (with

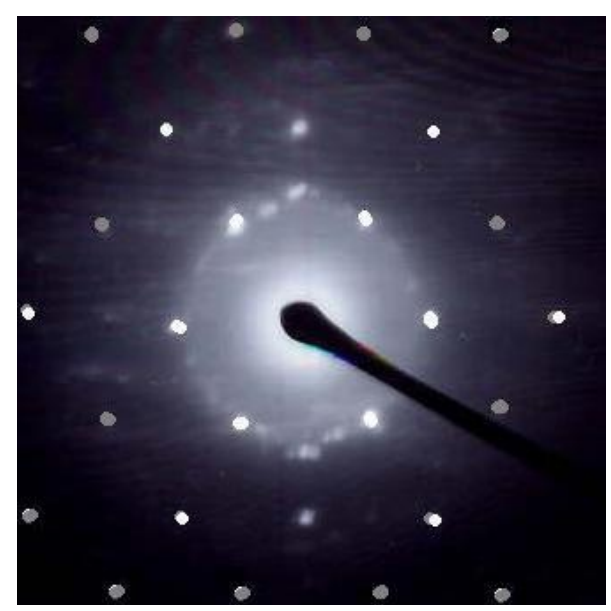

Figure 11. Electron diffraction pattern of the lubricant (para-ffin oil with oleic acid) layer on the monocrystalline carbon coating. 
and without additives) and may be advantageous for engineering practice as they improve antifriction characteristics of rubbing pairs and allow controlling the processes of boundary lubrication. Thus, received DLC monocrystalline coatings-orientants can be today the best materials of green tribology.

\section{References}

[1] Levchenko, V., Buyanovsky, I., Bolshakov, A., Matveenko, V. (2014) Green Tribology: Influence of New DLC Coatings-Orientants and Amorphous on Antifriction Properties of Lubricants. Journal of Electrical Engineering, 2, 39-48.

[2] Levchenko, V.A., Buyanovsky, I.A. and Drozdov, Yu.N. (2008) Modern Tribology: Results and Perspectives. LKI, Moscow, 480.

[3] Levchenko, V.A., Matveenko, V.N., Buyanovsky, I.A. and Ignatieva, Z.V. (2011) New Type of Nanocomposite Coatings. International Tribology Conference, Hiroshima, 30 October-3 November 2011, ITC D4-11.

[4] Drozdov, Yu.N., Buyanovsky, I.A., Levchenko, V.A., Matveenko, V.N., et al. (2014) Hard Carbon Coatings and Boundary Lubrication of Steel Parts. J. of Machinery Manufacture and Reliability, № 4, 298-305. https://doi.org/10.3103/S1052618814040049

[5] De Gennes, P.G. (1974) The Physics of Liquid Crystals. Oxford University Press, London, 330.

[6] Levchenko, V.A. (2000) Epitropic Liquid Crystals-A New Liquid Phase. Journal of Molecular Liquids, 85, 197-210. https://doi.org/10.1016/S0167-7322(99)00178-6

[7] Bol'shakov, A.N., Buyanovskii, I.A., Levchenko, V.A., Ignatieva, Z.V. and Matveenko, V.N. (2012) Laboratory Tribotests of Thin Carbon Coatings in Lubricants. Inorganic Materials, 48, 1359-1363. https://doi.org/10.1134/S0020168512140051

[8] Levchenko, V.A., Matveenko, V.N., Buyanovsky, I.A. and Ignatieva, Z.V. (2004) Influence of Carbon Coatings on Lubricating Properties of Boundary Layers. Journal of Engineering Tribology, 218, 485-493.

[9] Cognard, J. (1990) Tribology and the Liquid-Crystalline State. Proceedings of 198 National Meeting of the American Chemical Society, Miami Beach, Florida, 47.

[10] Levchenko, V., Buyanovsky, I., Zakharov, K., Bolshakov, A. and Matveenko, V. (2015) New Generation Carbon Coatings with Monocrystalline Structure as the Promising New Method of Oil Lubricity Increasing. Proceedings of Malaysian International Tribology Conference 2015, MYTRIBOS, Kuala Lumpur, 7-8.

[11] Beresaw, G. (1990) Tribology and the Liquid-Crystalline State. ASME Publishing, Washington DC, 135. https://doi.org/10.1021/bk-1990-0441

[12] Levchenko, V.A. and Popovskii, Yu.A. (2000) Orientational Ordering in 2,6-Lutidine near Quartz Surfaces Modified by Carbon. Journal of Molecular Liquids, 85, 211-217. https://doi.org/10.1016/S0167-7322(99)00179-8

[13] Levchenko, V.A., Buyanovskii, I.A., Ignatieva, Z.V., Bolshakov, A.N. and Matveenko, V.N. (2015) Antifriction Coating. RU Patent, 2570057.

[14] Buyanovskii, I.A., Levchenko, V.A., Bolshakov, A.N. and Matveenko, V.N. (2013) Effect of Structure and Composition of Solid Carbon Coatings of Steel Parts on the Lubricating Properties of Synthetic Oil. Journal of Friction and Wear, 34, 470-474. https://doi.org/10.3103/S1068366613050048 